\title{
Initial experience with new high powered I20 W holmium for vaporization of the prostate
}

\begin{abstract}
Holmium laser vaporization of the prostate (Holvap) is a minimally invasive treatment option available for men with urinary obstruction secondary to benign prostatic hyperplasia (BPH). Recently, a new $120 \mathrm{~W}$ high powered holmium laser has been approved to treat symptomatic prostatic obstruction (Lumenis Ltd). We present our early experience with the use of this laser. Prostatic configuration, uroflow patterns, post void residuals, and subjective AUA symptom score measurements were obtained and compared before and after treatment.
\end{abstract}

Volume 4 Issue 2 - 2017

\author{
Marc Beaghler, Mark Leo, Jaime Gass, Jordon \\ March, Sariely Sandoval, Alex Wonnaparhown \\ University of Nevada and San Buenaventura Community \\ Hospital, USA
}

Correspondence: Vaughan Daniels-Hepnar, University of Nevada and San Buenaventura Community Hospital, USA, Tel 44 75576555 23, Fax 972-4-9599070,

Email daniels.vaughan@lumenis.com

Received: September 16,2016 | Published: February 16, 2017

Abbreviations: $\mathrm{BPH}$, benign prostatic hyperplasia; TURP, transurethral resection of the prostate; LUTS, Lower urinary tract symptoms; PVR, post void residual; AUA, american urologic association

\section{Introduction}

There are several options currently available for treatment of symptomatic BPH including medications such as alpha-adrenergic blockers, or surgical options including monopolar or bipolar transurethral resection of the prostate (TURP), Greenlight laser vaporization (Nd:Yag) and holmium laser vaporization. ${ }^{1-6}$ In January 2015, a $120 \mathrm{~W}$ holmium laser system was released and approved for the treatment of BPH (Lumenis Pulse 120H). Since that time, nearly 100 patients have been enrolled in an ongoing prospective study with a complete dataset on 70 patients. The present study reports our initial experience using a high powered $120 \mathrm{~W}$ Holmium laser to treat lower urinary tract symptoms (LUTS).

The Pulse $120 \mathrm{H}$ offers technical advancements to both the laser and laser fiber representing an evolution from lower power holmium lasers of the past which were limited by longer operative times. ${ }^{7}$ In addition to providing higher energy output, the unit utilizes a foot pedal which allows the operator to easily alternate between laser vaporization and hemostatic settings without the use of a technician. Treatment with the $120 \mathrm{~W}$ holmium laser can be offered to patients with large prostate volumes, median lobe tissue, and to those in urinary retention. No patients in the current study were excluded from treatment due to high risk comorbidities or need for discontinuing ongoing anticoagulation therapy. Our experiences using the $120 \mathrm{~W}$ Holmium laser technologies demonstrate durable results comparable to published data using other surgical modalities for symptomatic BPH.

\section{Methods and materials}

A prospective ongoing study using the Pulse $120 \mathrm{H}$ holmium laser began in April 2015. Roughly 100 consenting men were evaluated for treatment of LUTS secondary to BPH. Patients were offered a variety of treatment options including conservative measures of oral medication and surgical options of standard TURP versus holmium laser TURP. The risks and benefits of each treatment option were presented and discussed.

Patients who opted for surgical options underwent cystoscopy, uroflowmetry, post void residual (PVR) bladder ultrasound, and American Urologic Association (AUA) symptom score questionnaires. PSA testing was also used when indicated. Those with cystoscopic evidence of prostatic hyperplasia, obstructive uroflowmetry pattern and Qmax values (max flow rate in $\mathrm{mL} / \mathrm{sec}$ ), incomplete bladder emptying with $>100 \mathrm{cc}$ post void residual, and moderate to severe AUA symptom score were considered candidates for treatment. Prostatic configuration, with the presence of middle lobe or median bar was not considered disqualifying factors for treatment with $120 \mathrm{~W}$ holmium lasers (Figure 1). Similarly, patients with multiple comorbidities who required continued anticoagulation therapy were treated. A total of thirteen patients were in urinary retention prior to treatment. Treatment with the holmium laser was performed as an outpatient procedure. Patients were discharged the same day with a two-way catheter in place and returned within 1-3 days of the postoperative period for a voiding trial. After removal of catheter, patients were seen for postoperative follow-up. At three months status post, all preoperative parameters were repeated. In addition to objective and subjective AUA symptoms score findings, patients were asked about their satisfaction with results of treatment. Surgeon satisfaction was also considered. 


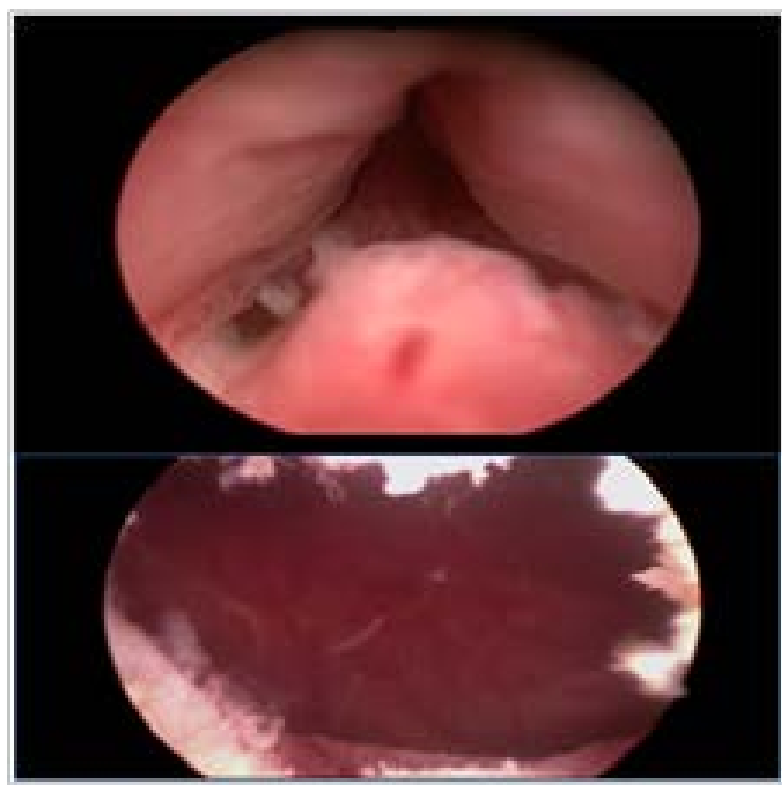

Figure I A before and after comparison using $120 \mathrm{~W}$ holmium laser for prostatic obstruction. A TURP-like defect is achieved upon completion.

\section{Surgical technique}

A standard technique was used for laser vaporization of the prostate. Normal saline was used as the irrigation fluid with a continuous flow cystoscope. Following induction of anesthesia, either a spinal or general anesthetic, careful evaluation of the prostate was performed to determine size and configuration. The Lumenis Pulse $120 \mathrm{H}$ was coupled with a side firing Xpeeda laser fiber and an increase to $120 \mathrm{~W}$ in total energy available. Using the laser fiber in a near contact mode, an incision was made at the 5 and 7 o'clock position in the prostate. The incision was performed distal to the ureteral orifice from the bladder neck to the verumontanum and was carried down to the level of the prostatic fibers. In a systematic fashion, the prostatic tissue was vaporized using a gentle sweeping motion. An attempt was made to develop a TURP-like defect. Throughout the procedure, the dual pedal system was utilized to rapidly alternate between vaporization and coagulation settings. For laser vaporization, standard settings of $2.0 \mathrm{~J}$ and $60 \mathrm{~Hz}$ were used. For hemostasis, the wide pulse option was utilized with lower standard settings of $1.0 \mathrm{~J}$ and $10 \mathrm{~Hz}$. These settings can be adjusted depending on desired effect on tissue. In general, we used higher power settings for vaporization and lower power and frequency for hemostasis. Hemostasis was achieved using only the holmium laser. No other technique, such as bipolar electrocautery was required.

\section{Results}

We have performed over 100 cases thus far using the Lumenis Pulse $120 \mathrm{H}$ and Xpeeda fiber with complete data on 70 patients. While we have noticed a slight learning curve to the system, intraoperative visualization and surgeon satisfaction has been excellent. The majority of patients' procedures was performed as outpatients and required only one fiber to be used per case. Most patients' catheters were removed by postoperative day three. Additionally, patients were treated without the requirement of hospital admission unless other comorbidities were present. Minor adverse events included postoperative irritative voiding symptoms, dysuria, and UTI, however these were self-limiting. A total of nine patients reported urinary frequency and four patients reported urinary urgency. There were very few major complications following surgery. These included a total of three episodes of urinary retention relieved with temporary placement of Foley catheter and two episodes of clot retention which required continuous bladder irrigation. One patient required retreatment with a standard TURP after 13 months due to urinary retention secondary to regrowth $\mathrm{BPH}$.

During the standardized three-month follow-up, patient satisfaction and objective measures were revisited (Table 1). The average preoperative AUA symptom score was 24.1 and decreased to 9.4 postoperatively. We saw similar improvements in average PVR from $175 \mathrm{cc}$ to $33 \mathrm{cc}$ with an average decrease of $142 \mathrm{cc}$. Qmax peak flow rates improved dramatically with an average increase of $7.1 \mathrm{cc}$ from $8.2 \mathrm{cc}$ to $15.3 \mathrm{cc}$. The average time of operation was 27 minutes and required $126 \mathrm{~kJ}$ of energy. Thirteen men in total were in retention (defined as PVR $>500 \mathrm{cc}$ or Qmax of $0 \mathrm{~mL} / \mathrm{sec}$ ) prior to treatment and all voided successfully following Holvap. Preoperative prostate volume was estimated for 32 out of the original 100 patients. Using both cystoscopy and ultrasound findings, the average volume was determined to be approximately 50 grams. The largest prostate treated was 166 grams.

Table I A comparison of pre and postoperative parameters repeated at three months status post.Values are presented as mean \pm standard deviation

\begin{tabular}{lll}
\hline Variable & Pre-OP & Post-OP \\
\hline Average AUA score & $24 . I \pm 8.19$ & $9.4 \pm 5.64$ \\
Average PVR (cc) & $175 \pm I 79.81$ & $33 \pm 38.52$ \\
Qmax $(\mathrm{mL} / \mathrm{sec})$ & $8.2 \pm 7.25$ & $15.3 \pm 9.4 \mid$ \\
\hline
\end{tabular}

\section{Discussion}

Early experiences with the $120 \mathrm{~W}$ holmium laser (Lumenis Ltd.) have demonstrated significant improvements in both subjective and objective parameters. In our opinion, the increase in overall power and advancements in the laser fiber technology provide greater hemostasis and a shorter operative time compared to prior versions. Nearly all cases were performed as an outpatient using a single fiber. Several patients with multiple medical comorbidities were treated successfully as well. While few adverse events have been reported, no patients have had any serious complications. The majority of patients were able to discontinue alpha-blocker and/or 5-alpha reductase inhibitor medications. Only one patient required retreatment with standard TURP after 13 months due to regrowth BPH. All 13 patients with preoperative urinary retention voided after treatment with laser vaporization.

We believe this new platform to be an evolution in technology. The non-exclusive nature of the $120 \mathrm{~W}$ holmium laser allows for treatment of a greater population including those with large glands, median lobe tissue, those in urinary retention, those on chronic anticoagulation therapy, and those not considered to be candidates for major surgery. ${ }^{2}$ Our results are comparable to published data using bipolar and standard TURP monopolar technology. ${ }^{7}$ We also compared these findings with our own personal experiences with the Greenlight laser for prostate vaporization. While similar improvements in AUA symptom score were seen in both modalities, holmium laser vaporization was found to confer a more significant decrease in post-void residual (142 vs 
$71 \mathrm{cc})$. Holmium and Greenlight laser vaporization were found to be similar in terms of post-operative complications and patient outcomes, however further randomized trials are needed to assess the overall differences between these modalities.

In terms of surgeon satisfaction, the option to alternate between vaporization and coagulation settings rapidly using the dual pedal system provided excellent hemostasis without the need for bipolar electrocautery or a dedicated technician..$^{8-11}$ Despite a higher power output, the $120 \mathrm{~W}$ holmium laser is notably quieter than previous $100 \mathrm{~W}$ systems. Another advantage of this technology is its multiple applications beyond soft tissue. In addition to vaporization and enucleation procedures, holmium is an ideal lithotripter for urinary calculi and thus provides a platform for the treatment of urolithiasis. ${ }^{10}$ While treatment with the holmium laser is not generally limited by prostatic configuration or anticoagulation therapy, patients with a prostate too large for standard or bipolar resection may require either holmium enucleation or open prostatectomy. ${ }^{3}$

\section{Acknowledgement}

None.

\section{Conflicts of interest}

The author declares there is no conflict of interest.

\section{References}

1. McVary KT, Roehrborn CG, Avins AL, et al. Update on AUA guideline on the management of benign prostatic hyperplasia. $J$ Urol. 2011;185(5):1793-1803.

2. Tan AH, Gilling PJ, Kennett KM, et al. Long term results of highpower holmium laser vaporization (ablation) of the Prostate. BJU Int 2003;92(7):707-709.
3. Moody JA, Lingeman JE. Holmium laser enucleation for prostate adenemoa greater than $100 \mathrm{gm}$ : comparison to open prostatectomy. $J$ Urolo. 2001;165(2):459-462.

4. Lee SW, Choi JB, Lee KS, et al. Transurethral procedures for lower urinary tract symptoms from benign prostatic enlargement: a quality and meta-analysis. Int Neruouol J. 2013;17(2):59-66.

5. Bouchier-Hayes DM, Van Appledorn S, Bugeja P, et al. A randomized trial of photoselective vaporization of the prostate using the $80-\mathrm{W}$ potassiumtitanyl-phosphate laser vs transurethral prostatectomy, with one year follow-up. BJU Int. 2010;105(7):964-969.

6. Teng J, Zhang D, Li Y, et al. Photoselective vaporization with the green light vs transurethral resection of the prostate for treating benign prostate hyperplasia: a systemic review and meta-analysis. BJU Int. 2013;111(2):312-323.

7. Mottet N, Anidjar M, Bourdon O, et al. A randomized Comparision of transurethral electroresection and holmium: Yag laser vaporization for symptomatic benign prostatic hyperplasia. J Endourol. 1999;13(2):227230 .

8. Delvecchio FC, Preminger GM. Endoscopic management of urologic disease with the holmium laser. Current Opinion in Urology. 2000;10(3):233-237.

9. Kumar A, Vasudeva P, Kumar N, et al. A prospective randomized comparative study of monopolar and photoselective vaporization of the prostate in patients who present with benign prostatic obstruction: a single center experience. J Endourol. 2013;27(10):1245-1253.

10. Grasso M. Experience with the holmium laser as an endoscopic lithotrite. Urology. 1996;48(2):199-206.

11. Beaghler M, Poon M, Ruckle H, et al. Complications employing the holmium: YAG laser. J Endourol. 1998;12(6):533-535. 